\title{
Steady state rheology from homogeneous and locally averaged simple shear simulations
}

\author{
Hao Shi ${ }^{1, \star}$, Stefan Luding ${ }^{1, \star \star}$, and Vanessa Magnanimo ${ }^{1, \star \star \star}$ \\ ${ }^{1}$ Multi Scale Mechanics, CTW, MESA+, University of Twente, 7500 AE Enschede, The Netherlands
}

\begin{abstract}
Granular materials and particulate matter are ubiquitous in our daily life and they display interesting bulk behaviors from static to dynamic, solid to fluid or gas like states, or even all these states together. To understand how the micro structure and inter-particle forces influence the macroscopic bulk behavior is still a great challenge today. This short paper presents stress controlled homogeneous simple shear results in a 3D cuboidal box using MercuryDPM software. An improved rheological model is proposed for macroscopic friction, volume fraction and coordination number as a function of inertial number and pressure. In addition, the results are compared with the locally averaged data from steady state shear bands in a split bottom ring shear cell and very good agreement is observed in low to intermediate inertia regime at various confining pressure but not for high inertia collisional granular flow.
\end{abstract}

\section{Introduction}

Granular media are envisaged as a collection of microscopic particles which interact through dissipative contact forces; their natural discontinuity poses many challenges for both academia and industry in understanding their bulk behavior [1]. A continuum description of the flow behavior of granular media is highly desirable, for application in both natural phenomena studies and industrial applications. Despite numerous efforts, the constitutive relations describing the granular flow behavior is still a matter of debate. Solid mechanics and kinetic theory have been successful in predicting the solid and gas like behavior, respectively [2,3]. At one extreme particles interact via enduring contacts, while in the gaseous regime, binary collisions are the mode of momentum exchange. The recently proposed inertial number framework has been successful in describing the flow behavior in the dense flow liquid like regime when the particles not only undergo collisions but also frictional interactions with other particles $[4,5]$. Though it very well predicts the flow behavior in case of steady shear of rigid particles, it fails in cases of inhomogeneous shear and transient shear, where we need better constitutive models to describe dense granular flow [6]. Also a non-local constitutive models have been proposed and tested to improve the constitutive relations in inhomogeneous flow [7]. With the development of computational power nowadays, one can obtain simulation data with sufficient statistics that allow to derive constitutive relations that describe the local rheology and flow behavior [8-12].

\footnotetext{
^e-mail: h.shi-1@utwente.nl

$\star \star$ e-mail: s.luding@utwente.nl

$\star \star \star$ e-mail: v.magnanimo@utwente.nl
}

A pressure density relation has been proposed in [13] in additive forms; the present paper proposes an extended local constitutive model in a multiplicative form and then tests the validity of this model for both homogeneous and inhomogeneous steady state soft granular flow.

\section{Simulation Setups}

\subsection{Stress controlled simple shear box}

We use MercuryDPM [14, 15], an open-source implementation of the Discrete Particle Method, to simulate a cuboid shear volume with Lees-Edwards periodic boundaries in $x$ and $y$ directions and normal periodic boundaries in $z$ direction. The particles are sheared along $x$ direction with a constant velocity $V_{x}$ and the stress is kept constant along $y$ direction as $\sigma_{y y}$. We vary the shear rate $\dot{\gamma}=\partial V_{x} / \partial y$ to understand the shear flow from quasi-static to dynamic regimes. The purpose of using Lees-Edwards periodic boundaries with constant stress is to represent dense granular flow in reality, e.g, sand or/and powders sheared in different shear cells or in an avalanche [10]. The piecewise linear elasto-plastic frictional contact model is used between particle contacts and the number of particles used in the simulation is 4096 . The other input parameters are summarized in Table 1.

\subsection{Split-Bottom ring shear cell}

The same MercuryDPM software was used to simulate a shear cell with annular geometry and a split bottom, as explained detailed in [16-18]. The geometry of the system consists of an outer cylinder (radius $R_{\mathrm{o}}=110 \mathrm{~mm}$ ) rotating around a fixed inner cylinder (radius $R_{\mathrm{i}}=14.7 \mathrm{~mm}$ ) with 
Table 1. Summary and numerical values of particle parameters used in the DEM simulations.

\begin{tabular}{llll}
\hline Parameter & Symbol & Simple Shear & Split-Bottom \\
\hline Average Diameter & $<r>$ & 2.2 and $2 \mathrm{~mm}$ & $2.2 \mathrm{~mm}$ \\
Polydispersity & $w=r_{\max } / r_{\min }$ & 2 and 3 & 2 \\
Particle Density & $\rho_{p}$ & $2000 \mathrm{~kg} / \mathrm{m}^{3}$ & $2000 \mathrm{~kg} / \mathrm{m}^{3}$ \\
Normal stiffness & $k$ & $10^{8} \mathrm{~kg} / \mathrm{s}^{2}$ & $100 \mathrm{~kg} / \mathrm{s}^{2}$ \\
Tangential stiffness & $k_{t} / k_{n}$ & 0.286 & 0.286 \\
Rolling/torsion stiffness & $k_{r} / k_{n}$ & 0.286 & 0.286 \\
Coulomb friction static & $\mu_{s}$ & 0.01 & 0.01 \\
Coulomb friction dynamic & $\mu_{d}$ & 0.01 & 0.01 \\
Rolling friction & $\mu_{r}$ & 0 & 0 \\
Restitution coefficient & $e$ & 0.804 & 0.469 \\
Normal Viscosity & $\gamma_{n}$ & $1 \mathrm{~kg} / \mathrm{s}$ & $0.002 \mathrm{~kg} / \mathrm{s}$ \\
Friction Viscosity & $\gamma_{t} / \gamma_{n}$ & 0.286 & 0.286 \\
Rolling Viscosity & $\gamma_{r} / \gamma_{n}$ & 0.286 & 0.286 \\
Torsion Viscosity & $\gamma_{r} / \gamma_{n}$ & 0.286 & 0.286 \\
\hline
\end{tabular}

a rotation frequencies of $\Omega=0.01$ to 0.75 revolutions per second. The granular material is confined by gravity between the two concentric cylinders, the bottom plate, and a free top surface. The bottom plate is split at radius $R_{\mathrm{S}}$ $=85 \mathrm{~mm}$. Due to the split at the bottom, a narrow shear band is formed. It moves inwards and widens towards the free surface. This set-up thus features a wide shear band away from the bottom and the walls, which is thus free from boundary effects. The filling height $(H \approx 40 \mathrm{~mm})$ is chosen such that the shear band does not reach the inner wall.

In earlier studies $[11,17]$, a quarter of this system $\left(0^{\circ}\right.$ $\leq \phi \leq 90^{\circ}$ ) was simulated using periodic boundary conditions. In order to save computation time, here we simulate only a smaller section of the system $\left(0^{\circ} \leq \phi \leq 30^{\circ}\right)$ with appropriate periodic boundary conditions in the angular coordinate, unless specified otherwise. No noticeable effect on the macroscopic flow behavior was observed in comparison between simulations done with a smaller $\left(30^{\circ}\right)$ and a larger $\left(90^{\circ}\right)$ angle.

\section{Dimensionless numbers}

The effects of varying shear rate, $\dot{\gamma}$, and confining pressure, $p$, can be modeled using two dimensionless numbers, expressed as a ratio of time-scales, as given in Table 2 , where $\rho_{p}$ is particle density and $k$ is the contact loading stiffness. Note that the subscripts in time scale ratio, $\dot{\gamma}, p$ and $k$ denote shear rate, pressure and contact duration respectively.

Table 2. Dimensionless numbers for the model

\begin{tabular}{lll}
\hline Dimensionless number & Definition & Time scale ratio \\
\hline Inertial number $I$ & $\frac{\dot{\gamma} d_{p}}{\sqrt{p / \rho_{p}}}$ & $t_{p} / t_{\dot{\gamma}}$ \\
Softness $p^{*}$ & $\frac{p d_{p}}{k}$ & $\left(t_{k} / t_{p}\right)^{2}$ \\
\hline
\end{tabular}

\section{Rheological model}

Here, a short summary of recent results on formulating an improved/accurate the local granular rheology is presented.

\subsection{Classical $\mu(I)$-rheology}

When formulating a granular rheology, the starting point is the successful, simple, and elegant so-called $\mu(I)$-rheology [5], which relates the so-called macroscopic (bulk) friction, i.e., the shear-stress to pressure ratio $\mu=\tau / p$, in a sheared particulate system, to the inertial number, $I$ :

$$
\mu(I)=\mu_{0}+\left(\mu_{\infty}-\mu_{0}\right) \frac{1}{1+I_{0} / I}
$$

This relation describes well a wide variety of flows [13] of hard, cohesionless particles, where $\mu_{0}=0.15$ and $\mu_{\infty}=0.42$ represent the zero and infinite strain-rate limits, respectively, and the characteristic dimensionless strainrate where the inertial effect kicks in, is $I_{0}=0.06$, .

\section{$4.2 \mu(I)$-rheology for soft particles}

The first improvement is based on particle softness, $p^{*}$, as introduced in $[11,18,19]$ :

$\mu\left(I, p^{*}\right)=\mu_{0} f_{\mu}(I) g_{\mu}\left(p^{*}\right)=\mu_{0}\left(1+\frac{\mu_{\infty} / \mu_{0}-1}{1+I_{\mu} / I}\right)\left(1-\left(\frac{p^{*}}{p_{\mu}^{*}}\right)^{0.5}\right)$

This two multiplicative corrections account for a range of strain rates and particle softness (or stiffness), but also for a system with a confined stress condition, as in the most shear rheometers, and even for different magnitudes of gravity, as in a centrifuge or on the moon [11]. Additional corrections for cohesive particles involve the socalled Bond-number $(\mathrm{Bo})$, as studied elsewhere [17] and ignored in the following. Apart from macroscopic friction, a rheological model has also been proposed for the bulk density (volume fraction) as a function of softness and shear rate:

$$
\phi\left(I, p^{*}\right)=\phi_{0} f_{\phi}(I) g_{\phi}\left(p^{*}\right)=\phi_{0}\left(1-\frac{I}{I_{\phi}}\right)\left(1+\frac{p^{*}}{p_{\phi}^{*}}\right)
$$

with the critical or steady state density under shear, in the limit of vanishing pressure and inertial number, $\phi_{0}$. The inertial number for which dilation turns to fluidization, $I_{\phi}$, and the typical pressure level for which softness leads to huge densities, $p_{\phi}^{*}[19]$. Note that both correction terms are valid only for sufficiently small arguments. Too large inertial numbers would fully fluidize the system so that the rheology should be that of a granular fluid, for which kinetic theory applies, while too large pressure would lead to enormous overlaps, for which the contact model and the particle simulation become questionable. In the following, the considered inertial numbers are $I<$ 0.5 , while the softness or pressure are $p^{*}<0.1$.

In order to complete the rheology for soft, compressible particles, a new relation for the coordination number, $C$, as function of pressure and shear rate is proposed here, analogous to Eqs. (2) and (3) (but data are not shown): 


$$
C\left(I, p^{*}\right)=C_{0} f_{C}(I) g_{C}\left(p^{*}\right)=C_{0}\left(1-\frac{I}{I_{C}}\right)\left(1+\left(\frac{p^{*}}{p_{C}^{*}}\right)^{\alpha}\right)
$$

with the critical or steady state coordination number under slow shear and low pressure, $C_{0}$, the inertial number order of magnitude for which dilation would turn to fluidization, $I_{C}$, the typical pressure level for which softness leads to a considerable increase in coordination number, $p_{C}^{*}$, and the power factor $\alpha$. Note that all multiplicative terms $(1 \pm \ldots)$ are first order Taylor expansions of possibly more realistic functions only, and $\mu_{0}, \phi_{0}$ and $C_{0}$ are constants but varying with different materials, i.e. when changing the inter-particle friction coefficient $\mu_{p}$.

\section{Results}

\subsection{Globally averaged rheology for simple shear}

For a system with very low inter-particle friction, $\mu_{p}=$ 0.01 , we simulated the stress controlled simple shear using the input parameters as shown in Table. 1, similar to Refs. $[11,12,17]$, except for the loading stiffness and restitution coefficient. We vary both shear rate $(\dot{\gamma}$ from 0.0001 to 1 $\mathrm{s}^{-1}$ ) and confining stress ( $p^{*}$ from 0.0025 to 0.1 ) to investigate the rate and softness dependencies. Unless specified, all the fitted functions shown in the following are using the data from homogeneous simple shear.

In Fig. 1, we plot the steady state bulk density (volume fraction) against the inertial number from our stress controlled homogeneous simple shear. Using the proposed $\phi\left(I, p^{*}\right)$ rheological function in Eq. (3), all our data are collapsing with the fitted curves; even for the highly inertial regime $(I>0.1)$ the bulk density is captured. However, if we check these curves focusing on only softness dependence as shown in Fig. 2, the lowest bulk density data (magenta) are actually deviating more from the fitting curve, and thus the low bulk density is not well captured.

In Fig. 2, we look at the confining stress dependence on the bulk density. When we keep the shear rate constant, the bulk density is increasing linearly with confining stress/pressure. Our data also fits well using the function from [11], which means our particle flow is also having a pressure dependence.

To check the quality of our fitting, we normalized our simple shear data using the fitted rheological model Eq. (3), $\phi\left(I, p^{*}\right)$. Up to $I$ around 0.1 , all the data points stay within $2 \%$ deviation range with no clear dependence on the inertial number, and this deviation increases slightly (still within 3\%) when the inertial number goes above 0.1 . However, the overall fitting quality is satisfactory (all within $\pm 3 \%$ ), and our rheological model is capturing well the bulk density of dense granular flow.

As for the macroscopic friction (Fig. 3), our improved rheological model is capturing well the friction behavior with deviation within $\pm 5 \%$. However, for coordination number (data not shown here), the deviation goes up to $\pm 25 \%$, which indicates a further improvement for Eq. (4) is still needed.

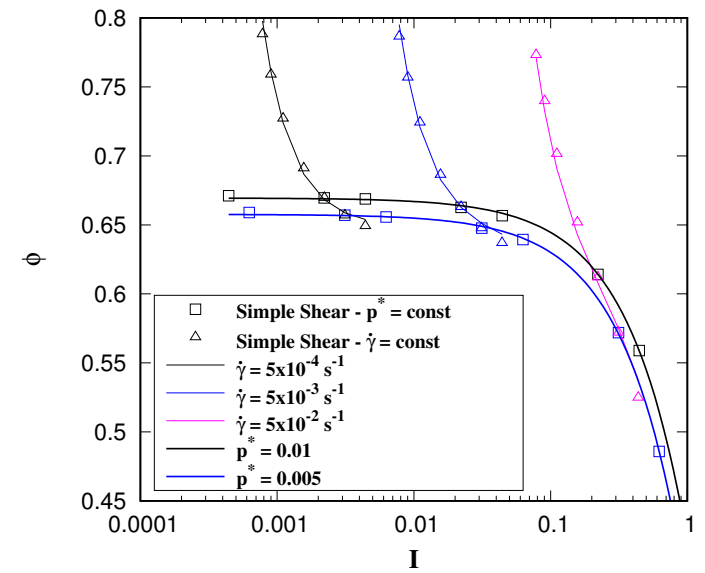

Figure 1. Bulk density, $\phi$, plotted against inertial number, $I$, using stress controlled simple shear. Two paths are chosen here: (i) softness $p^{*}=$ const., vary shear rate $\dot{\gamma}$; (ii) $\dot{\gamma}=$ const., vary softness $p^{*}$, as shown in the legend, different colors refer to different shear rates or confining stresses. The homogeneous data are fitted using $\phi\left(I, p^{*}\right)$, with $\phi_{0}=0.65, I_{\phi}=2.44$ and $p_{\phi}^{*}=0.38$.

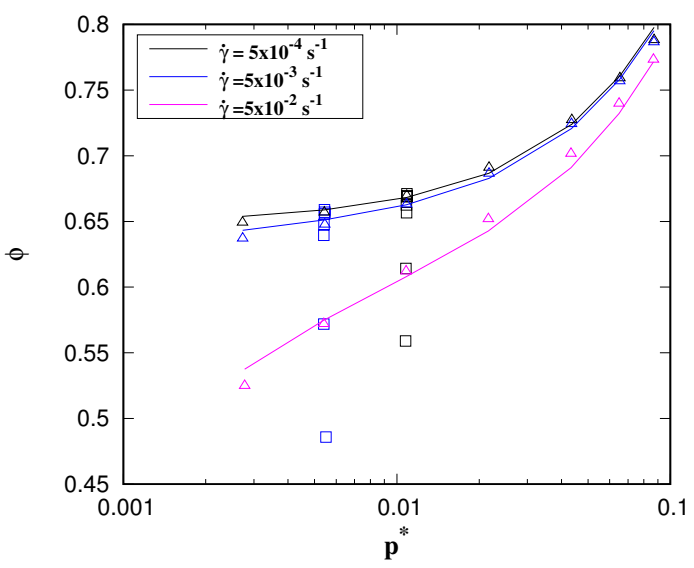

Figure 2. Bulk density, $\phi$, plotted against softness, $p^{*}$, for the same simulations as in Fig. 1. Note that here we neglect the fitting lines from path (i), which are vertical straight lines with constant $p^{*}$.

\subsection{Local rheology from split bottom ring shear cell}

The steady state bulk density from the center of shear bands in the split bottom ring shear cell are plotted on top of simple shear data as shown in Fig. 4. The split bottom shear cell data in low softness regime $\left(p^{*}<0.01\right)$ are collapsing almost perfectly with the simple shear data with only a slightly larger scattering except for high inertia regime $(I>0.01)$. For high softness regime $\left(p^{*}>0.01\right)$, the split bottom shear cell data are consistently deviating up to $+5 \%$ in all the investigated inertial numbers. And for the softness dependence as shown in Fig. 4(b), the deviation of split bottom ring shear cell data show the same increasing trend with increasing softness as simple shear data. The agreement between the two simulation setups is really good. 


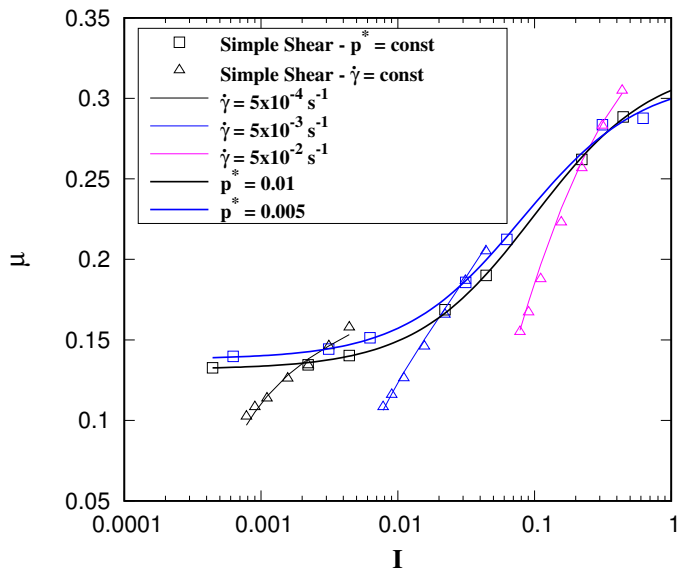

Figure 3. Macroscopic friction, $\mu=\tau^{*} / p^{*}$, plotted against inertial number, $I$, from the same simulations as in Fig. 1 . The data are fitted using $\mu\left(I, p^{*}\right)$ as Eq. (2), with $\mu_{0}=0.15, \mu_{\infty}=0.36$, $I_{\mu}=0.09$ and $p_{\mu}^{*}=0.60$.

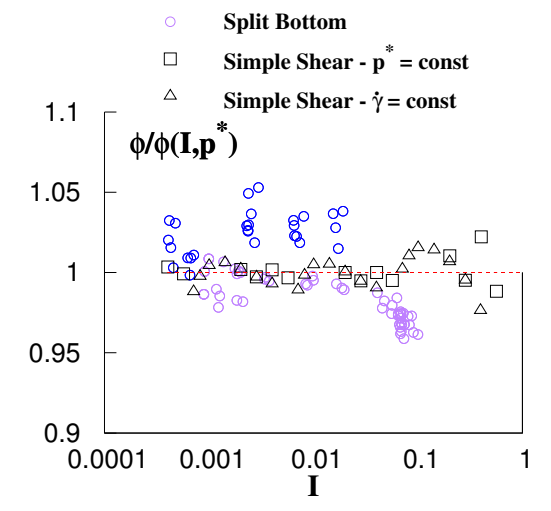

Figure 4. Bulk density comparison between simple shear and split bottom shear. The simulation data are normalized by the proposed rheology prediction $\phi\left(I, p^{*}\right)$ in Eq. (3). Blue and purple points are the split bottom shear cell data with softness $p^{*}>0.01$ and $p^{*}<0.01$ respectively.

\section{Conclusion}

The proposed rheological model for dense granular flow are checked using stress controlled simple shear simulations with looking at both inertial number and softness dependence. With increasing inertial number inside the system, the flow becomes more dilute, going towards collisional flow, where our model could not capture the behavior. Nevertheless, for granular flow with bulk density (volume fraction) above 0.5 and inertial number below 0.1 , our model gives very good bulk density and macroscopic friction descriptions of the influences both from inertia and softness in a sheared granular flow. Good agreement between the globally averaged simple shear box data and locally averaged center of shear band data inside a split bottom ring shear cell is achieved and further verifies the universality/applicability of the improved local rheological model. Future work will include the development of extending our model towards non-local inhomogeneous rheology as well as for cohesive systems.

\section{Acknowledgement}

We would like to thank for the financial support through the European-Union-Funded Marie Curie Initial Training Network FP7 "T-MAPPP" project (ITN607453).

\section{References}

[1] H.M. Jaeger, S.R. Nagel, R.P. Behringer, Rev. Mod. Phys. 68, 1259 (1996)

[2] I. Goldhirsch, Annu. Rev. Fluid Mech. 35, 267 (2003)

[3] R.M. Nedderman, Statics and kinematics of granular materials (Cambr. Univ. Press, Cambridge, 1992)

[4] B. Andreotti, Y. Forterre, O. Pouliquen, Granular media: between fluid and solid (Cambridge University Press, 2013)

[5] P. Jop, Y. Forterre, O. Pouliquen, Nature 441, 727 (2006)

[6] A. Ries, L. Brendel, D.E. Wolf, Comp. Part. Mech. pp. 1-8 (2015)

[7] M. Bouzid, M. Trulsson, P. Claudin, E. Clément, B. Andreotti, Phys. Rev. Lett. 111, 238301 (2013)

[8] S. Luding, Particulate Science and Technology 26, 33 (2007)

[9] S. Luding, F. Alonso-Marroquín, Granul. Matter. 13, 109 (2011)

[10] T. Weinhart, R. Hartkamp, A.R. Thornton, S. Luding, Physics of Fluids 25, 070605 (2013)

[11] A. Singh, V. Magnanimo, K. Saitoh, S. Luding, New J. Phys. 17, 043028 (2015)

[12] S. Roy, S. Luding, T. Weinhart, Procedia Engineering 102, 1531 (2015)

[13] S. Chialvo, J. Sun, S. Sundaresan, Phys. Rev. E 85, 021305 (2012)

[14] A. Thornton, T. Weinhart, S. Luding, O. Bokhove, Int. J. Mod. Phys. C 23, 1240014 (2012)

[15] T. Weinhart, A.R. Thornton, S. Luding, O. Bokhove, Granul. Matter. 14, 289 (2012)

[16] J.A. Dijksman, M. van Hecke, Soft Matter 6, 2901 (2010)

[17] A. Singh, V. Magnanimo, K. Saitoh, S. Luding, Phys. Rev. E 90, 022202+ (2014)

[18] S. Roy, S. Luding, T. Weinhart, arXiv preprint arXiv:1609.03098 (2016)

[19] S. Luding, A. Singh, S. Roy, D. Vescovi, T. Weinhart, V. Magnanimo, The 7th International Conference on Discrete Element Methods. (2016) 